\title{
Stability properties of a MIMO data flow controller
}

\author{
Ramón A. Delgado, Torbjörn Wigren, Katrina Lau and Richard H. Middleton
}

\begin{abstract}
The increasing demand for data in mobile applications has created a need for better communications systems. To satisfy this growing data demand next generation wireless systems are planned to use millimetre wave carrier frequencies. At these frequencies, radio shadowing is severe. To compensate for shadowing, incoming data flows must therefore be split and sent over several radio interfaces, that may have different delay properties. This paper analyses the stability properties of a MIMO data splitter that controls the delay skew between the data paths. The stability analysis is performed using IQC stability theory.
\end{abstract}

Index Terms-Control of networks, stability of nonlinear systems.

\section{INTRODUCTION}

Fifth generation $(5 \mathrm{G})$ wireless systems are expected to support several high-performance applications such as automotive safety, remote surgery and networked feedback control over wireless networks [1]. These applications require a wireless communication system with low latency and a constant sampling rate. Violation of these requirements may have severe consequences on performance and stability, as discussed in [2]. Current mobile broadband services have delays of tens of milliseconds, and large delay variations may occur. This fact makes them unsuitable for these new high-performance applications.

$5 \mathrm{G}$ wireless networks will operate at millimetre wave carrier frequencies. At these frequencies, radio wave propagation has beam-like behaviour where radio shadowing may be severe [1], [3]. The use of multi-point transmission may mitigate the effects of radio shadowing. Multi-point transmission increases the overall stability of the link by splitting incoming data into several data streams. These data streams are sent using multiple transmission nodes and are received at the other side of multiple wireless interfaces. The delays experienced by each data path may vary depending on the infrastructure and technologies used to transmit each data stream. Excessive delay skew in split flows can create problems with IP protocols, out of sequence packets, packet discard and retransmission. Thus, the delay skew between data streams needs to be controlled to reduce delay variation in the presence of radio shadowing.

In the companion paper [4] an algorithm for control of delay skew between data paths is proposed. This algorithm uses the data queues at the transmission nodes as actuators

\footnotetext{
R.A Delgado, K Lau and R.H. Middleton are with School of Electrical Engineering and Computing, University of Newcastle, Australia \{ramon.delgado, k.lau, richard.middleton\}@newcastle.edu.au

T. Wigren is with L5GR Systems, Ericsson AB, Stockholm, SE-16480 Sweden torbjorn.wigren@ericsson.com
}

to control the downlink delay experienced by each data path. This strategy ultimately allows the algorithm to control the downlink delay skew between several data paths. The present paper responds to the recently released call for more research on delays in networked control [5]. The main contribution of the paper is a MIMO stability analysis of the skew delay controller presented in the companion paper [4], and reviewed here for clarity of presentation. The analysis is performed using Integral Quadratic Constraints (IQC) theory [6].

Reference [4] and the current paper are part of an effort to tackle several delay control problems that arise in $4 \mathrm{G}$ and $5 \mathrm{G}$ wireless mobile communication networks. Related papers in this effort include [2], [4], [7], [8], [9]. In [8] the single input single output (SISO) inner loop used in this paper is described and analysed. Reference [7] describes an n-node round trip time skew control algorithm for critical machine type communications. The stability analysis in [7] combines IQC theory and high-order Padé approximations to provide a stability limit, in terms of the maximum allowed round trip loop delay. The main three aspects in which this paper differs from the work of [7] are (i) the control loop configuration, i.e. the block diagram and dynamics (ii) the handling of the time delays as operator components in the IQC theory, and (iii) the control objective that aims at controlling the downlink delay rather than the round trip delay. The paper [2] also treats a round trip delay skew control objective for the special case of two data paths. In this case, the stability problem is analysed by a combination of the SISO Nyquist and Popov criteria. The paper [9] analyses stability for the case of two data paths as well, but for the downlink delay skew control objective. The paper [4] is not focused on stability. It rather analyses the sensitivity and disturbance rejection properties of the algorithm of this paper, in order to provide general guidelines for design of the networked data flow control system. The present paper is thus restricted to stability analysis. Numerical results illustrating the performance of the proposed delay skew controller are available in [4].

The paper is organised as follows: Section II introduces the networked control architecture. The stability analysis is described in section III. Section IV presents a numerical example. Finally, conclusions are drawn in section V.

\section{Delay SKEW CONTROL}

This section reviews a controller that regulates the downlink delay skew between multiple data paths. The control scheme was presented in [9] for the two data path case with the $n+1$-data path version treated in the companion paper 
[4]. For completeness, and to introduce the notation for the IQC stability analysis, the description of the networked data flow controller is provided again here. Fig. 1 shows a block diagram of the MIMO downlink delay skew controller with $n+1$ transmission nodes and data paths. Cascade control is used, with a MIMO outer loop controller regulating the delay skew between data paths, and $n+1$ SISO inner loops controlling the queue dwell times.

\section{A. Outer loop}

The skew controller in Fig. 1 is located in the node where the data is split into multiple flows. The outer loop is composed of one delay sum control channel and $n$ delay skew control channels. The delay skew control channels regulate the $T_{\text {skew,i }}(s)$ to follow a reference signal $T_{\text {skew }, i}^{r e f}(s)$. One of the data paths is selected as the reference path and it is denoted by the subscript $r$. This data path is associated with the delay sum control channel. The sum control channel has a reference signal $T_{\text {sum }}^{r e f}(s)$, which sets the total delay budget available to be distributed between the $n+1$ data paths. The network interface uses some of this budget, but the remaining delay budget is available for distribution between the transmission node queues of the data streams. The total delay budget should be high enough to ensure that the control problem is feasible.

In the outer loop, the error signals $e_{i}(s), i=1, \ldots, n$ and $e_{r}(s)$ corresponding to the skew control channels and the sum control channel, respectively, are given by

$$
\begin{aligned}
e_{i}(s) & =T_{\text {skew }, i}^{\text {ref }}(s)-T_{\text {skew }, i}(s), \quad i=1, \ldots, n, \\
e_{r}(s) & =T_{\text {sum }}^{\text {ref }}(s)-T_{\text {sum }}(s) .
\end{aligned}
$$

The controllers $C_{\text {skew,i }}(s)$ and $C_{\text {sum }}(s)$ are used to compute the corresponding control actions $u_{i}(s), i=1, \ldots, n, r$ as follows

$$
\begin{aligned}
u_{i}(s) & =C_{\text {skew }, i}(s) e_{i}(s), \quad i=1, \ldots, n, \\
u_{r}(s) & =C_{\text {sum }}(s) e_{r}(s) .
\end{aligned}
$$

These control signals are then passed through a decoupling matrix $\mathbf{M}$, which enables $C_{\text {skew, }}(s)$ and $C_{\text {sum }}(s)$ to be designed using SISO techniques.

Static decoupling is performed using the matrix $M$. This decoupling matrix was derived in Theorem 1 in [7] and is given by

$$
\mathbf{M}=\frac{1}{n+1}\left(\begin{array}{cccccc}
n & -1 & \cdots & \cdots & -1 & 1 \\
-1 & n & -1 & \cdots & -1 & 1 \\
\vdots & \ddots & \ddots & \ddots & -1 & 1 \\
\vdots & \vdots & \ddots & \ddots & -1 & 1 \\
-1 & \cdots & \cdots & -1 & n & 1 \\
-1 & \cdots & \cdots & \cdots & -1 & 1
\end{array}\right) .
$$

The outputs of the static decoupling block are equal to the reference signals fed into the inner loops plus the downlink front haul delays.

The signals $T_{i}^{r e f}(s), i=1, \ldots, n, r$ are the reference signals for the inner loops. The inner loops are described as linear dynamic systems with transfer functions $G_{i}^{\text {inner }}(s)$, $i=1, \ldots, n, r$ in Fig. 1. The outputs of the inner loops are the dwell times $T_{i}(s)$ that are given as follows

$$
T_{i}(s)=G_{i}^{i n n e r}(s) T_{i}^{r e f}(s) \quad i=1, \ldots, n, r .
$$

The objective of the outer loop is to control the difference between data stream delays at the receiver location. Thus the signals $T_{i}(s)+T_{i}^{D L}(s), i=1, \ldots, n, r$ need to be formed before computing the delay skews. Next, these signals are transmitted to the controlling node. These transmissions are subject to uplink delays $T_{i}^{U L}, i=1, \ldots, n, r$. In this paper, it is assumed that the uplink delays are time invariant. Thus, the following assumption is assumed to hold.

A1) The uplink delays $T_{i}^{U L}, i=1, \ldots, n, r$ are time invariant.

This assumption is reasonable at least in the case when there is no congestion in the uplink. Assumption A1 allows the delay skews of the outer loop controller to be computed. The delay skews become

$$
\begin{aligned}
& T_{\text {skew }, i}(s)=e^{-s T_{i}^{U L}}\left(T_{i}(s)+T_{i}^{D L}(s)\right) \\
& -e^{-s T_{r}^{U L}}\left(T_{r}(s)+T_{r}^{D L}(s)\right), \quad i=1, \ldots, n, \\
& T_{\text {sum }}(s)=\sum_{i=1}^{n} e^{-s T_{i}^{U L}}\left(T_{i}(s)+T_{i}^{D L}(s)\right) \\
& +e^{-s T_{r}^{U L}}\left(T_{r}(s)+T_{r}^{D L}(s)\right) .
\end{aligned}
$$

The outer loop thus treats the uplink delays as constant signals, but it treats the downlink delays as dynamic signals.

\section{B. Inner loops}

The inner loop controllers apply feedback from the queue data volume. A block diagram of one of the inner loops is shown in Fig. 2. This inner loop has been studied previously in [8] and is also used in [4] and [9]. The reference for the inner feedback loop is obtained by transforming the dwell time reference signals $T_{i}^{r e f}(t), i=1, \ldots, n, r$ to data volume reference signals $y_{i}^{r e f}(t), i=1, \ldots, n, r$. This transformation is accomplished by multiplying the dwell time reference value $T_{i}^{r e f}(t)$ by the scheduled wireless data rate $w_{\text {air }, i}(t)$. Consistently, the queue dwell time $T_{i}(t)$ is obtained by dividing the queue data volume by the corresponding scheduled wireless data rate. To incorporate the fact that the network interfaces have limited capacity, and that the packets in the downlink are not sent in the uplink direction, a rate saturation is included in the inner loops.

Next, it needs to be assumed that the embedding in Fig. 2 is working as intended. This embedding allows cancelling the effect of $w_{\text {air }, i}(t)$ at the input and output of the inner loop. Assumptions A2 and A3 are introduced for this purpose, cf. [4].

A2) The bandwidth of $w_{a i r, i}(t)$ is significantly higher than the bandwidth of $T_{i}^{r e f}(t)$ for $i=1, \ldots, n, r$. The following relations therefore hold

$$
\text { 1) }\left(w_{\text {air }, i}(t)\right)^{-1} \approx\left(\bar{w}_{\text {air }, i}\right)^{-1}, i=1, \ldots, n, r \text {. }
$$




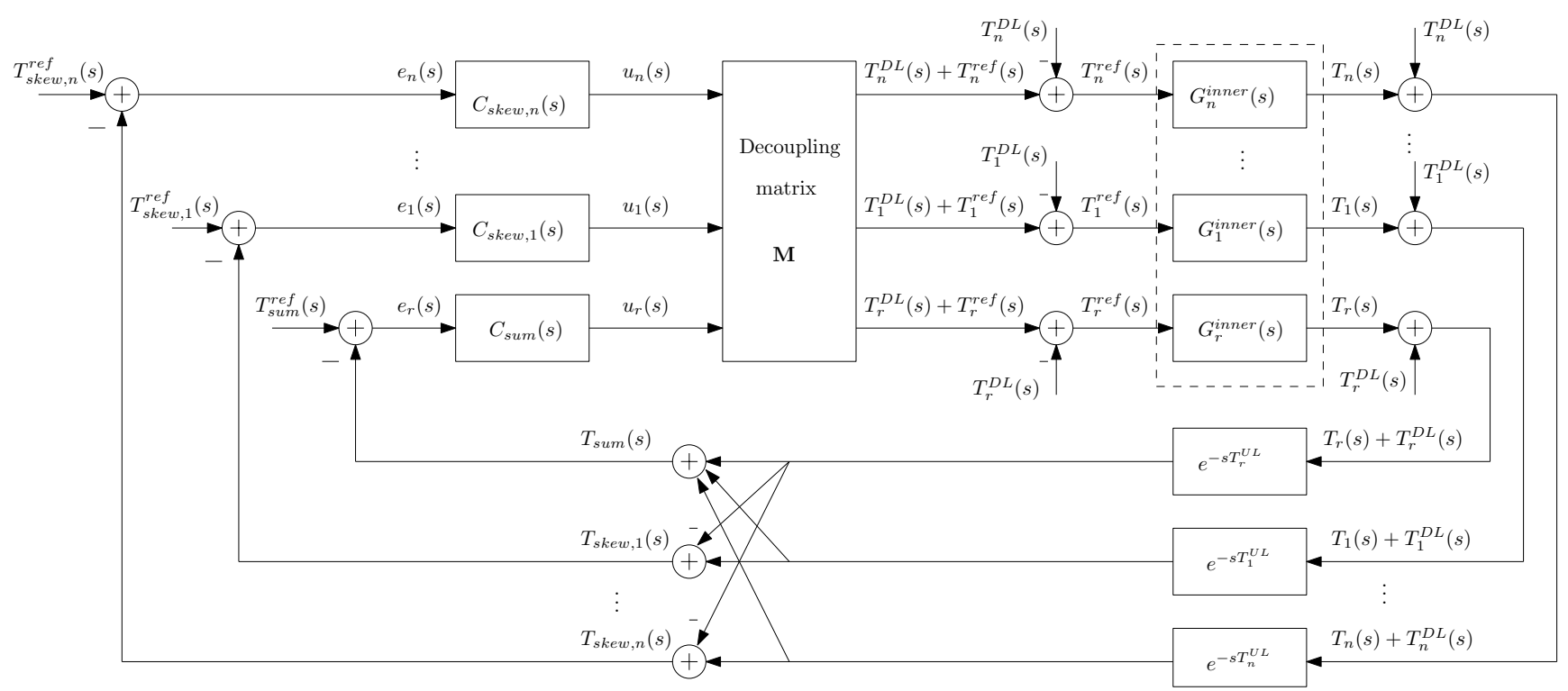

Fig. 1. Block diagram of the delay skew control system.

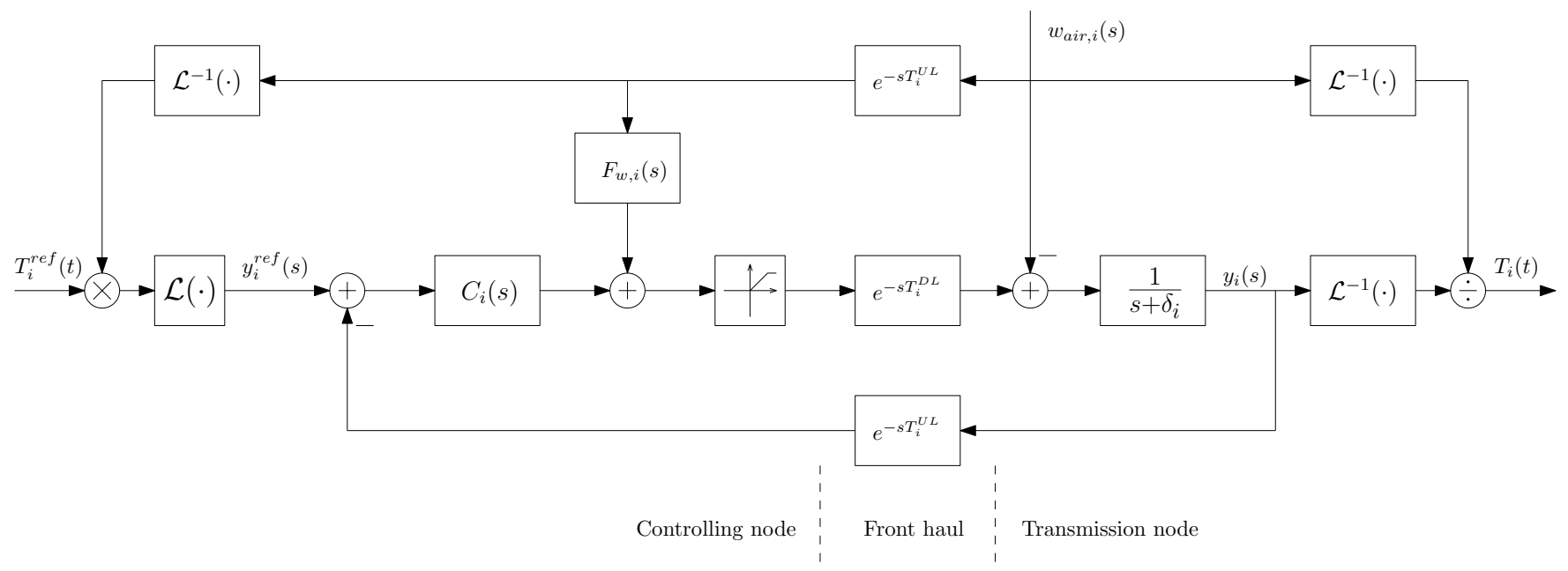

Fig. 2. Block diagram of one of the inner loops that controls the queue length.

2) $\mathcal{L}\left(T_{i}^{r e f}(t) w_{a i r, i}(t)\right) \approx T_{i}^{r e f}(s) \bar{w}_{a i r, i}, i=$ $1, \ldots, n, r$.

A3) The inner loops operate as intended, with data flow rate saturation being inactive.

Note that A3 is only assumed to cancel the effect of the embedding. The rate saturations are fully retained in the IQC analysis that follows below.

\section{MIMO model for stability analysis}

A prerequisite for the IQC stability analysis is to turn the block diagram in Fig. 1 into a standard representation. In [7] a similar approach was used. However, in [7], the control objective, inner loops and external signals were different. Hence a modified variant of the model is needed here. The IQC stability analysis is performed on a system having the following representation

$$
\begin{aligned}
\mathbf{v} & =\mathbf{G} \mathbf{w}+\mathbf{x} \\
\mathbf{w} & =\boldsymbol{\Delta} \mathbf{v} .
\end{aligned}
$$

$\mathbf{G}$ is a rational transfer function matrix with no poles in the right complex half plane. $\boldsymbol{\Delta}$ denotes a bounded causal operator, while $\mathbf{x}$ denotes an external vector signal. $\mathbf{v}$ and $\mathbf{w}$ denote the two internal vector signals.

The vector signal $\mathbf{v}(s)$ is given by

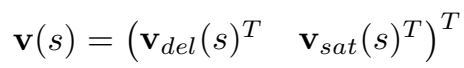

where

$$
\begin{aligned}
\mathbf{v}_{\text {del }}(s) & =\left(\begin{array}{llll}
v_{\text {del }, 1}(s) & \cdots & v_{\text {del }, n}(s) & v_{\text {del }, r}(s)
\end{array}\right)^{T} \\
\mathbf{v}_{\text {sat }}(s) & =\left(\begin{array}{llll}
v_{\text {sat }, 1}(s) & \cdots & v_{\text {sat }, n}(s) & v_{\text {sat }, r}(s)
\end{array}\right)^{T}
\end{aligned}
$$

Analogously, the signal $\mathbf{w}(s)$ is given by

$$
\mathbf{w}(s)=\left(\mathbf{w}_{\text {del }}(s)^{T} \quad \mathbf{w}_{\text {sat }}(s)^{T}\right)^{T}
$$


where

$$
\begin{aligned}
\mathbf{w}_{\text {del }}(s) & =\left(\begin{array}{llll}
w_{\text {del }, 1}(s) & \cdots & w_{\text {del }, n}(s) & w_{\text {del }, r}(s)
\end{array}\right)^{T} \\
\mathbf{w}_{\text {sat }}(s) & =\left(\begin{array}{llll}
w_{\text {sat }, 1}(s) & \cdots & w_{\text {sat }, n}(s) & w_{\text {sat }, r}(s)
\end{array}\right)^{T}
\end{aligned}
$$

The quantities of (11)-(16) appear in Fig. 3. The operator $\Delta$ accounts for the saturation and the delays in the inner loops. Thus, the bounded causal operator $\boldsymbol{\Delta}$ is composed as follows

$$
\Delta \mathbf{v}=\left(\begin{array}{cc}
\boldsymbol{\Delta}_{s a t} \mathbf{v}_{\text {sat }} & \mathbf{0} \\
\mathbf{0} & \boldsymbol{\Delta}_{\text {del }} \mathbf{v}_{d e l}
\end{array}\right)
$$

where

$$
\begin{gathered}
\boldsymbol{\Delta}_{\text {sat }} \mathbf{v}_{\text {sat }}= \\
\left(\begin{array}{cccc}
\operatorname{sat}_{1}\left(v_{\text {sat }, 1}(t)\right) & 0 & 0 & 0 \\
0 & \ddots & 0 & 0 \\
0 & 0 & \operatorname{sat}_{n}\left(v_{\text {sat }, n}(t)\right) & 0 \\
0 & 0 & 0 & \operatorname{sat}_{r}\left(v_{\text {sat }, r}(t)\right)
\end{array}\right)
\end{gathered}
$$

The part of $\Delta$ accounting for the delay is given by

$$
\left(\begin{array}{cccc}
v_{d e l, 1}\left(t-\tau_{1}(t)\right) & 0 & \boldsymbol{\Delta}_{\text {del }} \mathbf{v}_{\text {del }}= & \\
0 & \ddots & 0 & 0 \\
0 & 0 & v_{\text {del }, n}\left(t-\tau_{n}(t)\right) & 0 \\
0 & 0 & 0 & v_{\text {del, }, r}\left(t-\tau_{r}(t)\right)
\end{array}\right)
$$

where

where $\tau_{i}(t)=T_{i}^{D L}(t)+T_{i}^{U L}$. Fig. 3 shows a block diagram that is equivalent to the block diagram of Fig. 1. Notice that the inner loop controllers are now included in a diagonal MIMO controller $\mathbf{C}_{\text {inner }}(s)$ as follows

$$
\mathbf{C}_{\text {inner }}(s)=\left(\begin{array}{cccc}
C_{1}(s) & 0 & 0 & 0 \\
0 & \ddots & 0 & \vdots \\
\vdots & \ddots & C_{n}(s) & 0 \\
0 & 0 & 0 & C_{r}(s)
\end{array}\right)
$$

Similarly, $\mathbf{G}_{\text {queue }}(s)$ represents the MIMO model of the queues, i.e.

$$
\mathbf{G}_{\text {queue }}(s)=\left(\begin{array}{cccc}
\frac{1}{s+\delta_{1}} & 0 & 0 & 0 \\
0 & \ddots & 0 & \vdots \\
\vdots & \ddots & \frac{1}{s+\delta_{n}} & 0 \\
0 & 0 & 0 & \frac{1}{s+\delta_{r}}
\end{array}\right)
$$

The skew controllers are also included within a MIMO controller $\mathbf{C}_{\text {skew }}(s)$ as follows

$$
\mathbf{C}_{\text {skew }}(s)=\left(\begin{array}{cccc}
C_{\text {skew }, 1}(s) & 0 & 0 & 0 \\
0 & \ddots & 0 & \vdots \\
\vdots & \ddots & C_{\text {skew, } n}(s) & 0 \\
0 & 0 & 0 & C_{\text {sum }}(s)
\end{array}\right) .
$$

The computation of $T_{\text {skew, } i}(s), i=1, \ldots, n$, and $T_{\text {sum }}(s)$ as in (7)-(8) is now described by the matrix $\mathbf{F}$, which is

$$
\begin{gathered}
\mathbf{G}(s)= \\
\left(\begin{array}{cc}
\mathbf{0} & \left.-\mathbf{C}_{\text {inner }}(s)\left(\mathbf{M} \mathbf{C}_{\text {skew }}(s) \mathbf{F}+\mathbf{I}\right) \mathbf{G}_{\text {queue }}(s)\right) \\
\mathbf{I} & \mathbf{0}
\end{array}\right) \\
\mathbf{x}(s)=\left(\begin{array}{c}
\mathbf{x}_{1}(s) \\
0
\end{array}\right) \\
\mathbf{x}_{1}(s)=\mathbf{C}_{\text {inner }}(s) \mathbf{M} \mathbf{C}_{\text {skew }}(s) \mathbf{T}_{\text {skew }}^{\text {ref }}(s) \\
\\
\quad-\mathbf{C}_{\text {inner }}(s)\left(\mathbf{M C}_{\text {skew }}(s) \mathbf{F}+\mathbf{I}\right) \mathbf{T}^{D L}(s)
\end{gathered}
$$

$$
\mathbf{F}=\left(\begin{array}{ccccc}
1 & 0 & 0 & 0 & -1 \\
0 & \ddots & \ddots & \vdots & \vdots \\
\vdots & \ddots & 1 & 0 & -1 \\
0 & \cdots & 0 & 1 & -1 \\
1 & \cdots & 1 & 1 & 1
\end{array}\right)
$$

This allows the loop in the block diagram in Fig. 3 to be described in the form of (9)-(10) using$$
\mathbf{T}_{\text {skew }}^{\text {ref }}(s)=
$$$$
\left(T_{\text {skew }, 1}^{\text {ref }}(s) \quad \cdots \quad T_{\text {skew }, n}^{\text {ref }}(s) \quad T_{\text {sum }}^{\text {ref }}(s)\right)^{T}
$$$$
\mathbf{T}^{D L}(s)=
$$$$
\left(\begin{array}{llll}
T_{1}^{D L}(s) & \cdots & T_{n}^{D L}(s) & T_{r}^{D L}(s)
\end{array}\right)^{T}
$$

The block diagrams of Fig. 1 and Fig. 2 are now in the form of (9)-(10) required by the IQC analysis described in the following section.

\section{IQC STABILITY ANALYSIS}

This section presents an IQC stability analysis of the system defined in the previous section. The first step in the analysis is to review the basic tools and definitions used in IQC theory [6]. Next, assumptions on the components of the delay skew control scheme of Fig. 1 and Fig. 2 are introduced. Finally, the main result in IQC theory [6] is used to formulate Theorem 1 .

\section{A. Tools of Analysis}

The following definitions of [6], [10] are needed to set up the framework for the IQC analysis.

Definition 1: $\mathcal{L}_{2}^{m}$ denotes the space of $\mathcal{R}^{m}$-valued functions $f(\cdot):[0, \infty) \rightarrow \mathcal{R}^{m}$ of finite energy, i.e.

$$
\|f(\cdot)\|^{2}=\int_{0}^{\infty}|f(t)|^{2} d t<\infty .
$$

Definition 2: The space $\mathcal{L}_{2 e}^{m}$ is an extension of the space $\mathcal{L}_{2}^{m}$, whose members are $\mathcal{R}^{m}$-valued functions $f(\cdot)$ : $[0, \infty) \rightarrow \mathcal{R}^{m}$, such that their time truncation

$$
f_{T}(t)=\left\{\begin{array}{lr}
f(t), & 0 \leq t \leq T \\
0, & t>T
\end{array} \quad \in \mathcal{L}_{2}^{m} .\right.
$$

Definition 3: The feedback interconnection of $\mathbf{G}$ and $\boldsymbol{\Delta}$ as in (9)-(10) is well-posed if it defines a causal map $\mathbf{x} \rightarrow$ $(\mathbf{v}, \mathbf{w})$ on $\mathcal{L}_{2 e}$, i.e. for any $\mathbf{x} \in \mathcal{L}_{2 e}$ there exists a solution $(\mathbf{v}, \mathbf{w})$ that depends causally on $\mathbf{x}$. The interconnection is given by 


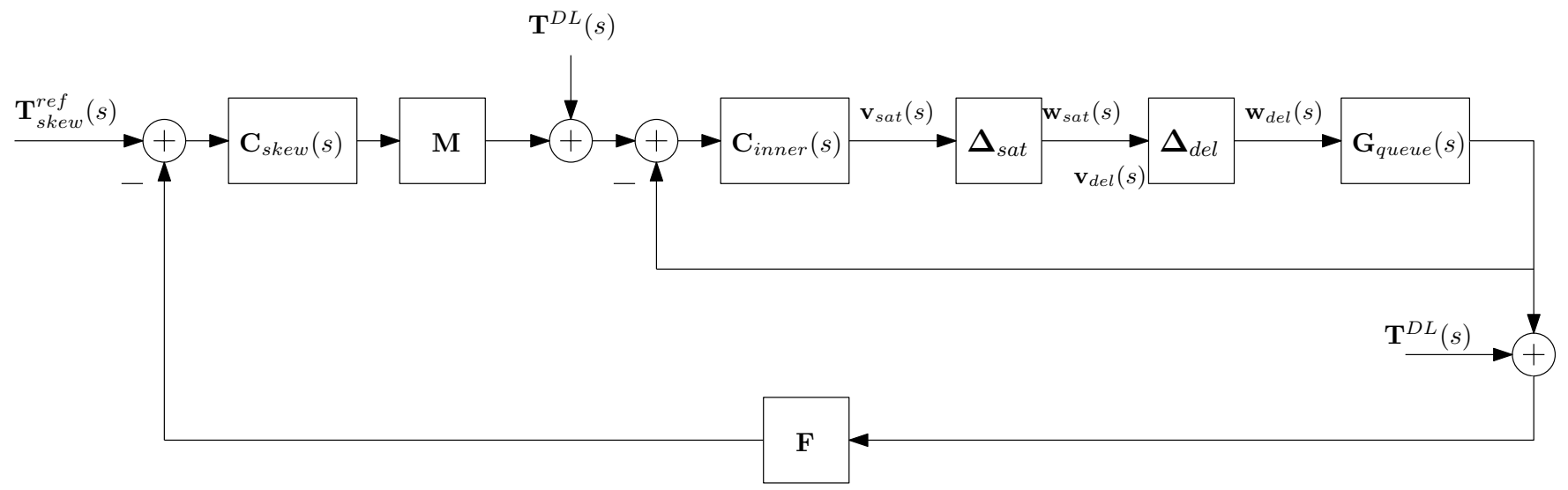

Fig. 3. Block diagram used in the IQC stability analysis.

stable if, in addition, the inverse is bounded. This means that there exists a constant $C_{I Q C}>0$ such that

$$
\int_{0}^{T}\left(|\mathbf{v}|^{2}+|\mathbf{w}|^{2}\right) d t \leq C_{I Q C} \int_{0}^{T}|\mathbf{x}|^{2} d t
$$

In the rest of the paper, the superscript $m$ in $\mathcal{L}_{2 e}^{m}$ will be omitted.

A bounded operator $\Delta$ is said to satisfy the IQC defined by $\Pi$, if for all $\mathbf{v}, \mathbf{w}$

$$
\int_{-\infty}^{\infty}\left[\begin{array}{c}
\mathbf{v}(j \omega) \\
\mathbf{w}(j \omega)
\end{array}\right]^{H} \boldsymbol{\Pi}(j \omega)\left[\begin{array}{c}
\mathbf{v}(j \omega) \\
\mathbf{w}(j \omega)
\end{array}\right] d \omega \geq 0
$$

with $\mathbf{w}=\Delta \mathbf{v}$. The matrix $\Pi$ is denoted the multiplier defining the IQC.

Next assume that the following conditions hold:

C1) $\quad \mathbf{G}$ is a proper rational function with real coefficients and without poles in the closed right half-plane.

C2) The interconnection of $\mathbf{G}$ and $\tau \boldsymbol{\Delta}$ is well posed for all $\tau \in[0,1]$.

C3) $\mathbf{x} \in \mathcal{L}_{2 e}$.

C4) $\Delta$ is a bounded causal operator.

C5) $\tau \boldsymbol{\Delta}$ satisfy the IQC defined by $\boldsymbol{\Pi}$.

The main result in IQC theory is then:

Lemma 1 ([6]): Assume that C1-C5 hold. If there exists $\epsilon>0$ such that $\forall \omega \in \mathcal{R} \cup\{\infty\}$

$$
\left[\begin{array}{c}
\mathbf{G}(j \omega) \\
I
\end{array}\right]^{H} \boldsymbol{\Pi}(j \omega)\left[\begin{array}{c}
\mathbf{G}(j \omega) \\
I
\end{array}\right] \leq-\epsilon I,
$$

then, the feedback interconnection of $\mathbf{G}$ and $\boldsymbol{\Delta}$ of (9)-(10) is stable.

A summary giving an intuitive explanation of C1-C5 appears in [7], c.f. [6] and [10].

\section{B. Assumptions on the delay skew controller}

In addition to assumptions A1-A3, the following assumptions are required for the IQC analysis of the delay skew controller.

A4) $\delta_{i}>0, i=1, \ldots, n, r$ and the transfer functions $C_{\text {sum }}(s), C_{r}(s), C_{\text {skew }, i}(s)$ and $C_{i}(s), i=$ $1, \ldots, n$ are proper rational transfer functions without poles in the closed right complex half-plane.
A5) $\mathbf{T}_{\text {ref }}^{\text {skew }}, \mathbf{T}^{D L} \in \mathcal{L}_{2 e}$

A6) The interconnection of $\mathbf{G}$ and $\tau \Delta$ is well posed for every $\tau \in[0,1]$.

Assumption A4 ensures that $\mathbf{G}$ is a proper rational transfer function with real coefficients without poles in the right half-plane. The assumption that $\delta_{i}>0$ for $i=1, \ldots, n, r$ indicates that Active Queue Management [11] is used in the inner loops. The assumption on the location of the poles of the controllers indicates that such controllers must be open loop stable.

\section{Verification of IQC conditions}

Before applying the IQC result described in Lemma 1 it is necessary to verify that assumptions A1-A6 imply that C1-C5 hold. Condition C1 follows from (19) if $\mathbf{C}_{\text {skew }}(s)$, $\mathbf{C}_{\text {inner }}(s)$ and $\mathbf{G}_{\text {queue }}(s)$ are proper rational transfer functions without poles in the closed right half-plane. Then, assumption A6 ensures that $\mathrm{C} 1$ holds. Assumption A4 ensures that $\mathbf{C}_{\text {skew }}(s)$ and $\mathbf{C}_{\text {inner }}(s)$ are bounded operators, thus A4-A5 ensure that condition C3 is met. From the analysis in [6], [10] it is well known that conditions C4-C5 are satisfied by the bounded operators considered in the current paper. Assumptions A1-A3 are needed to represent the cascade block diagram in Figures 1 and 2 into the representation (9)-(10) used for IQC stability analysis. Assumption A3 was made with the purpose to make the embedding in Fig. 2 possible and not with the purpose to ignore the sector nonlinearities in the stability analysis. These nonlinearities are now included in the stability analysis to give the following result.

Theorem 1: Consider the feedback interaction of (9)-(10) and assume that the conditions A1-A6 hold. Suppose that $\boldsymbol{\Delta}$ of (17) satisfy the IQC given by $\boldsymbol{\Pi}$. Then, the feedback interconnection (9)-(10) is stable if there exists $\epsilon>0$ such that (26) holds $\forall \omega \in \mathcal{R} \cup\{\infty\}$.

\section{NumERicAl RESUlTS}

To illustrate the analysis above, numerical stability results are given. Proportional controllers are first chosen for the inner and outer loop. This choice of the controllers provides insights into the trade-offs in the controller design. The IQC stability tests were performed using the IQC toolbox [12]. 


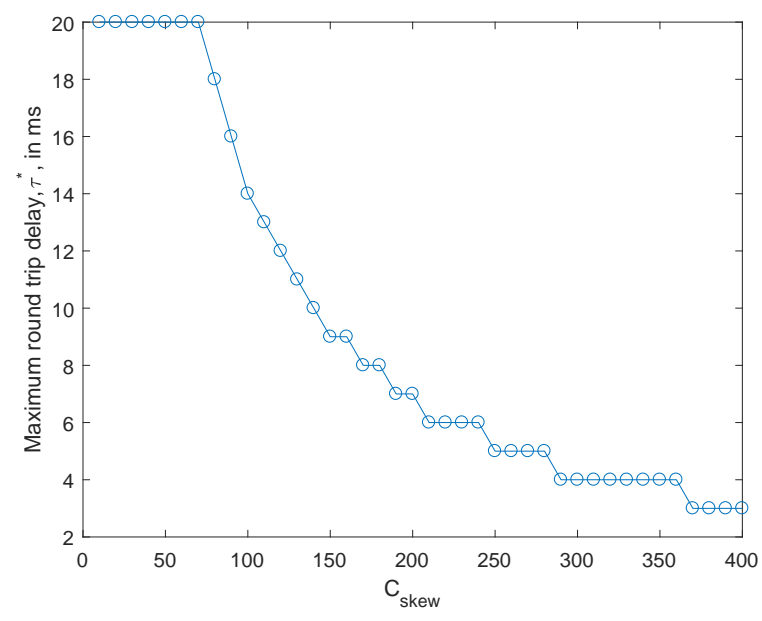

Fig. 4. Maximum round trip delay which guarantees stability, $\tau^{*} \in$ [1 $1 \mathrm{~ms}, 20 \mathrm{~ms}]$, as a function of $C_{\text {inner }}$.

For the numerical analysis it is assumed that a saturation satisfies the IQC of a sector nonlinearity and also the IQC given by the Popov multiplier. Stability is tested under the premise that each delay satisfies the IQCs for an uncertain time-invariant delay, see [6], [12]. The IQC stability tests search for the maximum round-trip delay, $\tau^{*}$, which implies stability of the MIMO control loop. First, consider the case in which there are three data paths, i.e. $n=2$, that $\mathbf{C}_{\text {skew }}=I_{3}$ and $\mathbf{C}_{\text {inner }}=C_{\text {inner }} I_{3}$ where $C_{\text {inner }}$ is a constant gain. Fig. 4 shows the maximum round trip delay, $\tau^{*} \in[1 \mathrm{~ms}, 20 \mathrm{~ms}]$, that guarantees stability as a function of $C_{\text {inner }}$. In Fig. 4 $C_{\text {inner }}$ varies between 10 and 400 . Note that when $C_{\text {inner }} \leq$ 70 stability is guaranteed for $\tau \leq 20 \mathrm{~ms}$.

Next, consider the case in which there are two data paths, i.e. $n=1$, that $\mathbf{C}_{\text {skew }}(s)=C_{\text {skew }}(s) I_{2}$ and $\mathbf{C}_{\text {inner }}(s)=$ $C_{\text {inner }}(s) I_{2}$ where

$$
\begin{aligned}
C_{\text {skew }}(s) & =0.1 \frac{s+5.76}{10 s+5.76}, \\
C_{\text {inner }}(s) & =\alpha \frac{0.22798(s+30.49)(s+2.3)}{(s+69.51)(s+0.23)},
\end{aligned}
$$

and $\alpha$ is the low-frequency gain of $C_{\text {inner }}(s)$, i.e. $\alpha=$ $C_{\text {inner }}(0)$. Note that $C_{\text {skew }}(0)=0.1$. The controllers in (27)-(28) have the poles and zeros used in [9], but the static gains are different. IQC stability tests were performed using $\alpha \in[10,20]$. Fig. 5 shows $\tau^{*}$ as a function of $\alpha$. The value $\tau=-1$ denotes that there is no stability guarantee. In Fig. 5 when $C_{\text {inner }}(0) \leq 16$ stability is guaranteed for $\tau \leq 20 \mathrm{~ms}$, but if $C_{\text {inner }}(0) \geq 18$ then there is no stability guarantee using the IQC analysis proposed here. This result is explained by [3].

\section{CONCLUSION}

The use of multi-point transmissions will compensate the adverse shadowing conditions at millimetre wave carrier frequencies. A synchronised arrival of originally adjacent data packets sent over these flows is crucial for the success of the multi-point transmission strategy. This paper analyses

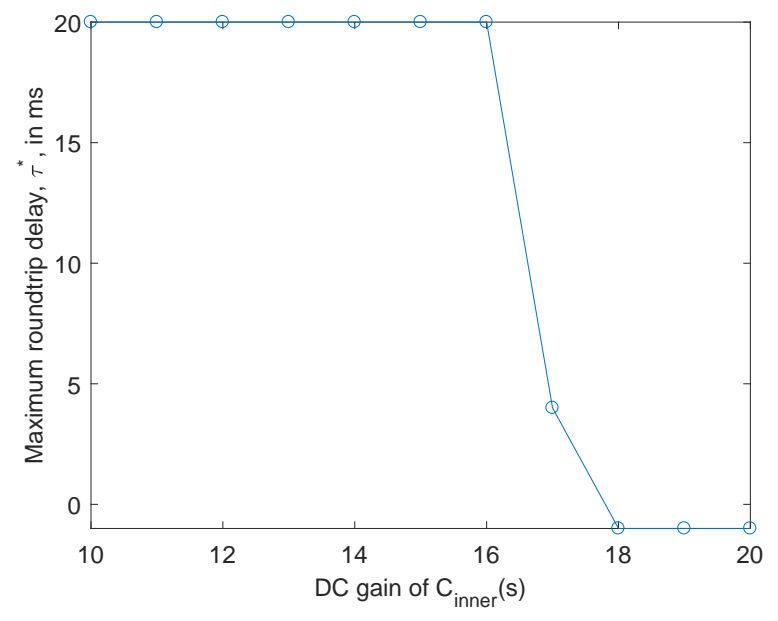

Fig. 5. Maximum round trip delay which guarantees stability, $\tau^{*} \in$ $[1 \mathrm{~ms}, 20 \mathrm{~ms}]$, as a function of the low-frequency gain of $C_{i n n e r}(s)$.

the stability of a recently proposed downlink delay skew controller. The stability analysis of this MIMO controller is performed using IQC theory. The numerical results are consistent with experimental test bed results reported in a companion paper and related publications.

\section{ACKNOWLEDGEMENTS}

This research was supported under Australian Research Council's Linkage Projects funding scheme (project number LP150100757).

\section{REFERENCES}

[1] T. S. Rappaport, R. W. H. Jr., R. C. Daniels, and J. N. Murdock, Millimeter Wave Wireless Communications. Prentice Hall, 2014.

[2] R. H. Middleton, T. Wigren, K. Lau, and R. A. Delgado, "Data flow delay equalization for feedback control application using 5G wireless dual connectivity," to appear in Proc. VTC2017-Spring, Sydney, Australia, June 2017.

[3] T. Wigren, "Low-frequency limitations in saturated and delayed networked control," in Proc. IEEE CCA 2015, Sydney, Australia, Sep. 21-23 2015 .

[4] K. Lau, T. Wigren, R. A. Delgado, and R. H. Middleton, "Disturbance rejection properties for a $5 \mathrm{G}$ networked data flow delay controller," submitted to CDC 2017, Melbourne, Australia, 2017.

[5] T. Samad, "Control systems and the internet of things," IEEE Control Systems Magazine, vol. 36, pp. 13-16, 2016.

[6] A. Megretski and A. Rantzer, "System analysis via integral quadratic constraints," IEEE Trans. Automat. Contr., vol. 42, no. 6, pp. 819-830, 1997.

[7] R. A. Delgado, K. Lau, R. H. Middleton, and T. Wigren, "Networked delay control for $5 \mathrm{G}$ wireless machine type communications using multi-connectivity," submitted to IEEE Trans. Contr. Sys. Tech., 2016.

[8] T. Wigren, "Robust $\mathcal{L}_{2}$ stable networked control of wireless packet queues over delayed internet connections," IEEE Trans. Contr. Syst. Tech., vol. 24, pp. 502-513, 2016.

[9] T. Wigren, K. Lau, R. A. Delgado, and R. H. Middleton, "Delay skew packet flow control in 5G wireless systems with dual connectivity," submitted to IEEE Trans. Vehicular. Tech., 2017.

[10] C.-Y. Kao and A. Rantzer, "Stability analysis of systems with uncertain time-varying delays," Automatica, vol. 43, pp. 959-979, 2007.

[11] R. Adams, "Active queue management: a survey," IEEE Comm. Surveys \& Tutorials, vol. 15, pp. 1425-1476, 2013.

[12] C.-Y. Kao, A. Megretski, U. T. Jonsson, and A. Rantzer, "A MATLAB toolbox for robustness analysis," in Computer Aided Control Systems Design, IEEE International Symposium on, Taipei, 2004, pp. 297-302. 